\title{
Interleukin 10 Production Correlates with Pathology in Human Leishmania donovani Infections
}

\author{
Hashim W. Ghalib, " Marcia R. Piuvezam, ${ }^{\ddagger}$ Yasir A. W. Skeiky, ${ }^{\ddagger}$ Mohamed Siddig, ${ }^{3}$ Faisal A. Hashim, \\ Ahmed M. El-Hassan, ${ }^{*}$ Donna M. Russo, ${ }^{*}$ and Steven G. Reed ${ }^{\ddagger}$ \\ ${ }^{*}$ University of Juba, College of Medicine, Khartoum, Sudan; ${ }^{\ddagger}$ Seattle Biomedical Research Institute, Seattle, Washington 98109 ; \\ and ${ }^{\S}$ University of Khartoum, College of Medicine, Khartoum, Sudan
}

\begin{abstract}
We have found that an important Th2 cytokine, IL-10, is produced by tissues from patients acutely infected with Leishmania donovani. In all individuals tested, IL-10 mRNA production was increased in lymph nodes taken during acute disease over that observed in postacute samples. In contrast, both preand posttreatment lymph nodes had readily detected mRNA for IFN- $\gamma$ and IL-2. A down-regulating effect of IL-10 on leishmania-induced proliferative responses was demonstrated when Hu rIL-10 was added to cultures of PBMC from clinically cured individuals. PBMC from individuals with acute visceral leishmaniasis responded to stimulation with leishmania lysate by producing IL-10 mRNA. Simultaneously cultured PBMC collected from the same patients after successful chemotherapy produced no detectable IL-10 mRNA after leishmania antigen stimulation. Neutralizing anti-IL-10 mAb added to PBMC from patients with acute visceral leishmaniasis markedly increased the proliferative response to leishmania lysate. Finally, we observed mRNA for IL-10 and IFN- $\gamma$ concurrently in a lesion from a patient with post-kala-azar dermal leishmaniasis (PKDL). These results indicate the production of IL-10 during L. donovani infection, and suggest a role for this cytokine in the regulation of immune responsiveness during visceral leishmaniasis. (J. Clin. Invest. 1993. 92:324-329.) Key words: leishmaniasis • interleukin 10 - Leishmania donovani • cytokines
\end{abstract}

\section{Introduction}

Leishmania are protozoan parasites of macrophages and are thus in a position to both influence and be influenced by host cytokines. One such cytokine, IL-10, is of particular interest because of its antagonistic effects on IFN- $\gamma$, a potent activator of macrophages for intracellular killing of leishmania (1). The production of IL-10 could influence the outcome of infection by partially blocking production of $\operatorname{IFN}-\gamma(2,3)$. Perhaps more importantly, IL-10 also has powerful effects on macrophages. These include down-regulation of antigen presentation $(4,5)$, cytokine production $(6,7)$, and of metabolic events associated with macrophage activation (8). Thus, an important role of IL-10 in parasitic infections may be its ability to block the

Address correspondence to Dr. Steven G. Reed, Seattle Biomedical Research Institute, 4 Nickerson Street, Seattle, WA 98109.

Received for publication 22 December 1992 and in revised form 10 February 1993.

J. Clin. Invest.

(C) The American Society for Clinical Investigation, Inc.

0021-9738/93/07/0324/06 \$2.00

Volume 92, July 1993, 324-329
IFN-mediated activation of macrophages to kill intracellular protozoa (9).

Human visceral leishmaniasis, caused by $L$. donovani or $L$. infantum in Africa, India, and Europe, or by $L$. chagasi in Latin America, is most often recognized as an acute infection with severe morbidity and high mortality in untreated cases. These patients are characterized immunologically as having high levels of anti-leishmania antibody and low or absent leishmania-elicited T cell proliferation and cytokine (IL-2 and IFN$\gamma$ ) production, responses which become positive upon recovery from acute disease, (10-12). Delayed hypersensitivity responses to leishmania antigens parallel the in vitro lymphocyte response patterns $(13,14)$.

The classification of murine CD4+ $\mathrm{T}$ cells into those producing Th 1 cytokines and those producing Th 2 cytokines ( 15 , 16) has proven to be a major advance in the elucidation of mechanisms of resistance and susceptibility to experimental leishmaniasis as well as other parasitic infections (reviewed in 17). In murine leishmaniasis, the Th 2 cytokines IL- 4 and IL10 have been associated with susceptibility to visceralizing cutaneous $L$. major infections $(18,19)$. The production of active IL-10 has also been associated with genetic susceptibility to Trypanosoma cruzi in mice (9). In both of these models, the Th 1 cytokine IFN- $\gamma$ is the one that has been most closely associated with healing responses $(18,20)$.

Cytokine production patterns in human visceral leishmaniasis, particularly with regard to the production of down-regulating Th2 cytokines, have not been well documented. Of particular interest is the production of cytokine or cytokine mRNA in lymphoid tissue, as well as in leukocytes of the peripheral circulation. In the present study, we have determined that an important Th2 cytokine, IL-10, is produced during human infections with $L$. donovani and provide evidence for a role for IL-10 in regulating aspects of T cell responses in visceral leishmaniasis patients.

\section{Methods}

Patient material. Patients with visceral leishmaniasis and post-kalaazar dermal leishmaniasis (PKDL) were seen at the University of Khartoum Soba hospital. Active visceral leishmaniasis was identified clinically by fever of more than two-wk duration, hepatosplenomegaly, and pancytopenia. Diagnosis was confirmed by the identification of leishmania amastigotes in impression smears obtained from the lymph node, bone marrow, and/or spleen. Epitrochlear lymph nodes were excised from patients available for both pre- and posttreatment evaluation following informed consent. Lymph nodes were snap frozen for subsequent freeze sectioning. Peripheral blood samples or lymph nodes were obtained before and $30 \mathrm{~d}$ after, completion of treatment with

1. Abbreviations used in this paper: PKDL, post-kala-azar dermal leishmaniasis; SI, stimulation index. 
sodium stibogluconate (Pentostam; Wellcome Reagent Ltd., London, UK) at $10 \mathrm{mg} / \mathrm{kg}$ body wt i.v.

PKDL patients. Patient 1 had PKDL for $10 \mathrm{yr}$ following a subclinical infection with visceral leishmaniasis. The patient had depigmented papular and maculopapular eruptions in the face, limbs, and trunk. A diagnosis of PKDL was confirmed by the finding of leishmania amastigotes in slit smears and in histological sections of lesions. The pathology of skin sections showed a mononuclear cell reaction consisting of lymphocytes, plasma cells, and macrophages, many of which contained amastigotes. PBMC and frozen skin biopsy of a maculopapular lesion were obtained. The patient was successfully treated with Pentostam at $20 \mathrm{mg} / \mathrm{kg}$ body wt for $30 \mathrm{~d}$. Patient 2 had active visceral leishmaniasis 6 yr previously. He was treated with Pentostam at $10 \mathrm{mg} / \mathrm{kg}$ daily for $14 \mathrm{~d}$, and responded well with resolution of acute disease symptoms. 2 mo after treatment he developed papular, nodular lesions and infiltration plaques in the face, neck, trunk, and upper limbs. Leishmania amastigotes were found in slit smears and in sections of the lesions. The pathology showed well organized epithelioid granulomas and lymphocytes in the dermis.

PBMC culture and proliferation assays. PBMC were separated by Ficoll-Hypaque (Winthrop Laboratories, New York), frozen, and, together with the frozen skin biopsies, lymph nodes, and sera, were carried to Seattle for analyses. For proliferation assays, PBMC $\left(3 \times 10^{5}\right.$ cells/well) were cultured in complete medium (RPMI 1640 supplemented with gentamycin, 2-ME, L-glutamine, and $10 \%$ screened pooled A+ human serum; Trimar, Hollywood, CA) in 96-well flat bottom plates with or without $20 \mu \mathrm{g}$ protein $/ \mathrm{ml}$ of total cell lysate prepared from culture promastigotes of $L$. donovani (World Health Organization designated reference strain MHOM/ET/67/HU3) or 5 $\mu \mathrm{g} / \mathrm{ml}$ of PHA (Sigma Immunochemicals, St. Louis, MO) for $5 \mathrm{~d}$. The cells were pulsed with $1 \mu \mathrm{Ci}$ of $\left[{ }^{3} \mathrm{H}\right]$ thymidine (ICN Immunochemicals, Costa Mesa, CA) for the final $18 \mathrm{~h}$ of culture and incorporation of label was assessed by liquid scintillation counting. Data are represented as mean cpm of triplicate cultures and stimulation index (SI), defined as mean cpm of cultures with parasite lysate/mean cpm of cultures without lysate. To assess the role of IL-10 in lymphocyte proliferative responses, PBMC from acute or postacute visceral leishmaniasis patients were cultured with anti-IL-10 $(50 \mu \mathrm{g} / \mathrm{ml})$ or Hu rIL-10 $(2 \mathrm{ng} /$ $\mathrm{ml}$ ) and in the presence or absence of parasite lysate.

Cytokine PCR. Total RNA was isolated from PBMC cultured as above at $2 \times 10^{6}$ cells/well, or frozen lymph node sections, 40 for each specimen, by the guanidinium thiocyanate phenol chloroform extraction (21) and cDNA was synthesized using poly ( $\mathrm{dT}$ ) (Pharmacia LKB Biotechnology, Piscataway, $\mathrm{NJ}$ ) and $\mathrm{RNase}^{-}$reverse transcriptase (Gibco BRL Life Technologies, Inc., Gaithersburg, MD) in a final reaction volume of $20 \mu$ l. Samples were brought to $200 \mu$ l with water. cDNA (following normalization to B-actin) were amplified by PCR with AmpliTaq DNA polymerase (Perkin Elmer Cetus Corp., Norwalk, CT) with $0.2 \mu \mathrm{M}$ of the respective $5^{\prime}$ and $3^{\prime}$ external primers in a volume of $50 \mu \mathrm{l}$. For a semi-quantitative estimation of cytokine mRNA, the initial $20 \mu \mathrm{L}$ cDNA synthesis reaction was adjusted to final dilutions of 1:20,1:100, and 1:500. The primer pairs used and the PCR conditions were from published information (22). 25-30 cycles were used for PCR.

Cytokine probes. Plasmids containing the human sequences IL-2, IL-4, IFN- $\gamma$ (23), and B-actin (no. 65128; American Type Culture Collection, Rockville, MD) were digested with HindIII/EcoRI, EcoRI, SacI/HindIII, and EcoRI respectively. Human IL-10 cDNA was cloned by PCR from mitogen-stimulated PBMC from normal donors using oligonucleotide primers designed to amplify a 535 base pair fragment spanning the entire coding region of HuIL-10 (24). The cDNA was subcloned into P-Bluescript and digested with BamHI/EcoRI for isolation of insert. DNA fragments were excised from agarose gels, electroeluted, and purified as described (25). Radiolabeled ${ }^{32} \mathrm{P}$-probes were prepared by random priming (26).

Southern analysis. PCR products were analyzed by electrophoresis on $1.5 \%$ agarose gels, transferred to nylon membranes, and probed with the appropriate ${ }^{32} \mathrm{P}$-labeled DNA insert. Hybridization was performed at $55^{\circ} \mathrm{C}$ overnight. Posthybridization washes were at $55^{\circ} \mathrm{C}$ for $20 \mathrm{~min}$ twice each with $2 \times$, and $1 \times$ SSC containing $0.2 \%$ SDS.

Reagents. Hu rIL-10 and neutralizing anti-Hu IL-10 (JES3-19F1) were gifts of DNAX Research Institute, Palo Alto, CA. Both reagents had $<0.1 \mathrm{U}$ of endotoxin.

\section{Results}

Production of IL-10 by lymph nodes from visceral leishmaniasis patients before and after chemotherapy. Acute visceral leishmaniasis patients are leukopenic, but have hyperplasia of secondary lymphoid organs. Thus problems such as selective depletion and altered trafficking of PBMC could influence results obtained by studying these cells. To obtain a more complete picture of the cytokine response during acute and postacute disease, we examined lymph nodes taken from four individuals before and after a standard regimen of Pentostam chemotherapy of 30-d duration, as well as from uninfected US donors. Leishmania were detected in sections of lymph nodes taken before, but not after treatment (not shown). A semi-quantitative PCR for select cytokines was performed on frozen sections of lymph nodes. To normalize for total tissue and $T$ cell mRNA, control PCR were performed using B actin and CD3. To verify that $P C R$ was performed within the range of linearity, serial dilutions of cDNA were used in the PCR, which resulted in corresponding decreases in PCR product.

In comparing lymph node cytokine patterns between acute and convalescent patients, striking differences were observed in the expression of IL-10 mRNA, which was present in lymph nodes from all four individuals with acute visceral leishmaniasis. In contrast, little or no IL-10 mRNA was detected in nodes from the same patients following successful chemotherapy (Fig. 1). Differential expression of IL-4 mRNA was detected in acute vs. posttreatment lymph nodes of two patients (Fig. 1, patients 3 and 4). However, no IL-4 mRNA was detected in nodes from one patient, and no difference was seen in comparative samples from another. Message for these cytokines was not detected in lymph nodes from uninfected individuals. These results indicated that lymphoid tissue RNA expression of a major Th2 T cell cytokine, IL-10, and, to a lesser extent IL-4, was elevated in acute visceral leishmaniasis.

Comparative analyses of $\mathrm{Th} 1$ cytokine mRNA production were performed on the same lymph node sections. IFN- $\gamma$ mRNA was detected in visceral leishmaniasis patient lymph nodes both before and after treatment, with no apparent differences in amounts at the two time points. IL-2 mRNA, although present in most of the pretreatment samples, appeared to be more abundant after treatment in three of the four patients. Thus there was not a clear pattern of differential expression of these Th1 cytokine productions between acute and cured patients; in general, mRNA for both IL- 2 and IFN- $\gamma$ was expressed in lymph nodes from both stages of infection.

Effects of IL-10 on the in vitro proliferation of visceral leishmaniasis patient PBMC. IL-10 has been described as having several types of down-regulating effects on immune responses. Because of the finding that abundant IL-10 mRNA was present in acute but not in postacute patient lymph nodes and sera, we examined the possibility that IL- 10 could have a role in the lack of antigen-driven proliferative responses traditionally associated with acute visceral leishmaniasis.

PBMC from three individuals with postacute visceral leishmaniasis were cultured with or without $L$. donovani lysate, in 


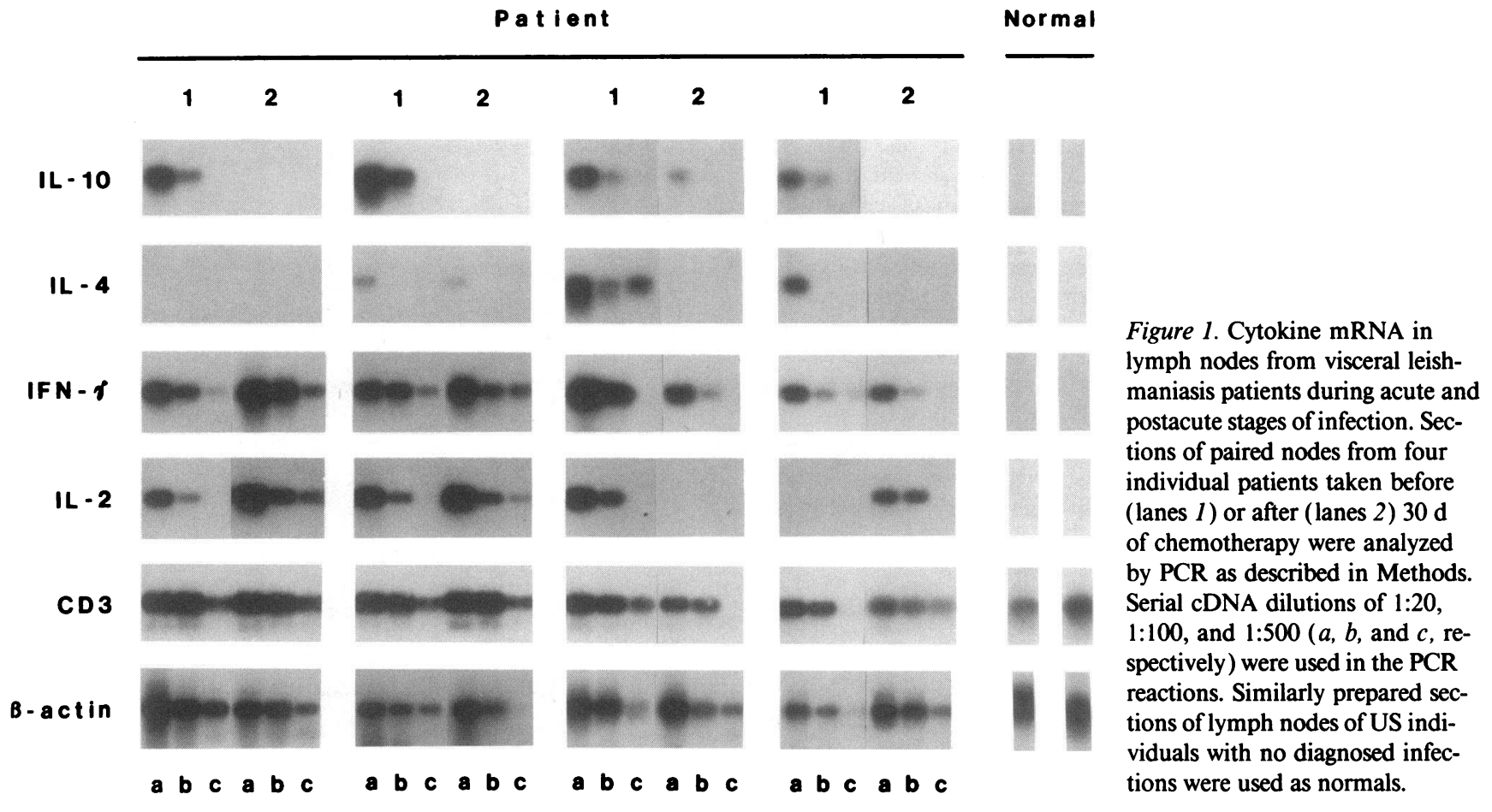

the presence or absence of human rIL-10, in a standard proliferation assay. As expected, the PBMC proliferated in response to leishmania lysate (Fig. 2). However, the addition of Hu rIL-10 to the cultures completely blocked proliferative responses in all individuals tested, and even reduced background proliferation. This observation provided evidence that IL-10 could play a role in down-regulating antigen-driven PBMC proliferation in leishmaniasis patients.

IL-10 mRNA production by PBMC from visceral leishmaniasis patients. Previous studies have documented that PBMC from patients with active visceral leishmaniasis do not proliferate when cultured with crude leishmanial antigen preparations. This has led to the conclusion that PBMC from acute patients are not responsive to leishmanial antigen, while PBMC proliferative responses can be elicited upon resolution of acute infec-

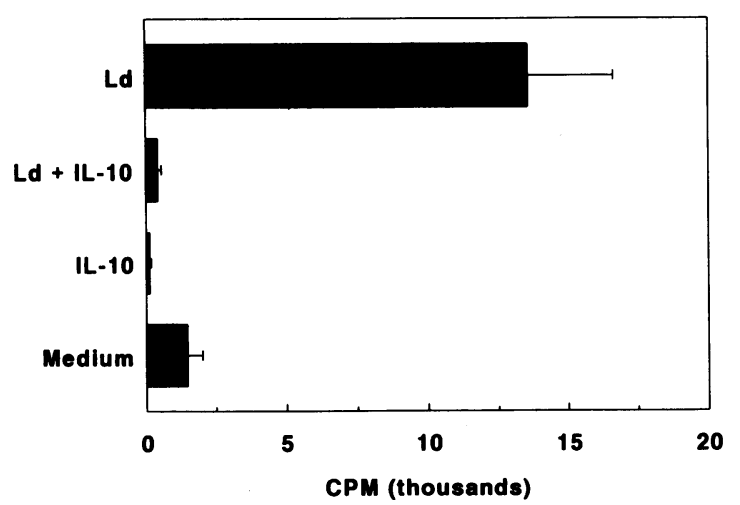

Figure 2. IL-10 inhibits proliferative responses of PBMC from convalescent visceral leishmaniasis patients. Cells were obtained from individuals 3-6 mo after treatment for visceral leishmaniasis. Cells were cultured for $5 \mathrm{~d}$ with $L$. donovani lysate in the presence or absence of $2 \mathrm{ng} / \mathrm{ml} \mathrm{Hu}$ rIL-10. Results from 5 patients are shown as mean cpm (SEM) of triplicate cultures.

tion. We used PCR to examine cytokine mRNA production in leishmania-stimulated and unstimulated PBMC from patients with active and cured visceral leishmaniasis. PBMC from three patients, not the same individuals used in Fig. 1, were collected both during acute disease and $3 \mathrm{wk}$ later, after completing a course of chemotherapy. PBMC from a fourth patient were collected during acute disease only. Cells were cultured with or without $L$. donovani lysate, and cytokine PCR performed.

PBMC from all of the acute phase patients produced IL-10 mRNA in response to leishmania lysate (Fig. 3). Maximum IL-10 mRNA production was obtained $48 \mathrm{~h}$ after antigen stimulation (other time points not shown). PBMC cultured in the absence of leishmania lysate did not produce IL-10 mRNA. In one individual, IL-10 mRNA was detected in PBMC not cultured in vitro, although apparently increased IL-10 mRNA was also observed in this individual following stimulation with leishmania lysate. In contrast, none of the PBMC collected after chemotherapy produced IL-10 mRNA, with or without antigenic stimulation. These results demonstrate that PBMC from patients with acute visceral leishmaniasis can, in fact, respond to leishmania antigen(s). This response differs from that of cells from convalescent individuals in that it results in the production of IL-10 mRNA rather than proliferation or production of IL-2 and IFN- $\gamma$ mRNA. As expected, the opposite was observed with regard to IL-2 mRNA production. PBMC from convalescent, but not from acute patients, had IL-2 mRNA whether or not they were stimulated with leishmania lysate (not shown). These results correlated with leishmania-driven proliferative responses observed in paired PBMC samples from four patients. No proliferation was seen in acute patient PBMC (SI 1.3 \pm 0.04 ), while responses were positive following treatment ( SI 12.4 \pm 2.1 ), as has been previously documented (10).

Anti-IL-10 increases the proliferation of PBMC from acute visceral leishmaniasis patients. The production of IL-10 by antigen-stimulated PBMC could be important in decreasing 


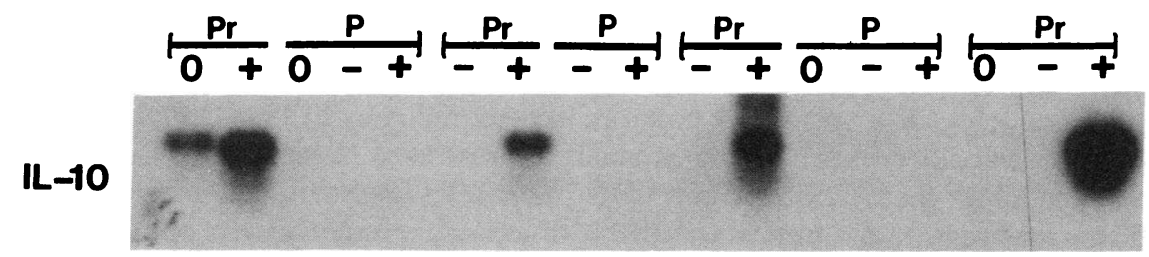

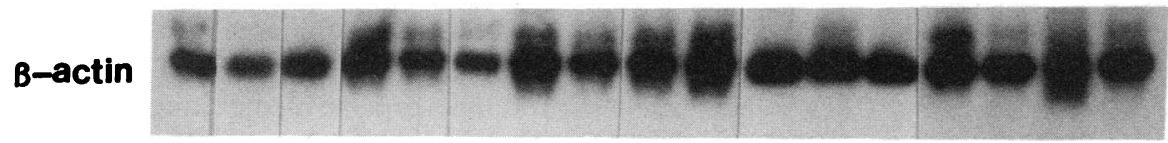

Figure 3. IL-10 mRNA production by acute visceral leishmaniasis patient PBMC in response to leishmania. PBMC from three patients were examined during acute infections $(P r)$ and following $30 \mathrm{~d}$ of chemotherapy $(P)$. PBMC from a fourth patient were examined befnre treatment only ( $P r$, right $)$. PBMC were cultured as described in Methods, and prepared for PCR at the initiation of culture (lanes 0 ), or after $48 \mathrm{~h}$ of culture without (lanes -) or with (lanes +) L. donovani lysate. Unstimulated or leishmania lysate-stimulate PBMC from uninfected individuals produced no detectable IL-10 mRNA (not shown). proliferative responses in visceral leishmaniasis patients. To examine this, PBMC from patients with acute visceral leishmaniasis were cultured with leishmania lysate and a neutralizing $\mathrm{mAb}$. The cells proliferated in the presence of the combination of leishmania lysate and anti-IL-10 mAb, but not to either the mAb or lysate alone (Fig. 4). An isotype matched control $\mathrm{mAb}$ had no effect on proliferation. Results from this experiment and from the PCR studies illustrated in Fig. 3 indicate that antigen-induced IL-10 production by visceral leishmaniasis patient PBMC may be responsible, at least in part, for the lack of leishmania-specific proliferative responses of these cells in vitro.

Cytokine production in PKDL. Nonulcerative cutaneous lesions may develop in both Indian and African visceral leishmaniasis following resolution of acute disease. The cytokine profile of either the lesions or the PBMC from these patients has not been described. PBMC from two PKDL patients as well as a lesion biopsy from one such individual, were examined for the production of IL-10 and IFN- $\gamma$ mRNA. Both individuals' PBMC produced mRNA for IFN- $\gamma$, but not for IL-10, upon in vitro stimulation with leishmania lysate (Fig. 5). Unstimulated cells did not produce mRNA for either cytokine. Lesion biopsy tissue from one of the PKDL patients was analyzed for cytokine mRNA by PCR, and found to have strong expression of IFN- $\gamma$, IL-2, IL-4, and IL-10. Thus, a PKDL lesion expressed

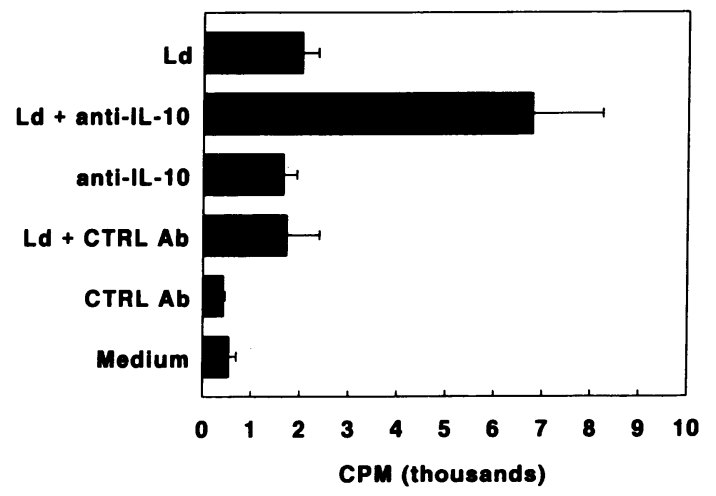

Figure 4. Neutralizing anti-IL-10 mAb restores the proliferative response of acute visceral leishmaniasis patient PBMC. Cells were cultured for $5 \mathrm{~d}$ with $L$. donovani lysate with or without $50 \mu \mathrm{g} / \mathrm{ml}$ antiIL-10 mAb or isotype-matched control mAb. Data are mean cpm (SEM) of triplicate cultures, from 3 individual patients. both Th1 and Th 2 cytokines, although PBMC from the same individual expressed only Th1 among the cytokines examined. No detectable PCR products for these cytokines were detected in normal skin biopsies (not shown).

\section{Discussion}

Studies in mouse models of parasitic infection have associated the two general groups of $\mathrm{T}$ cell cytokines with key regulatory roles in determining disease outcome. IL-10 is reportedly produced by genetically susceptible, but not resistant, mice infected with either L. major or T. cruzi, and this important Th2 cytokine is unique in that it can decrease both the production and effective utilization of IFN- $\gamma$, a Th 1 cytokine. IFN- $\gamma$ is the cytokine most closely associated with resistance to infection with protozoan parasites. Macrophages can be effectively activated by IFN- $\gamma$ to kill leishmania in vitro, and presumably in vivo as well $(27,28)$. Macrophages are the exclusive host cell for leishmania in the mammalian host. Therefore, cytokines

A

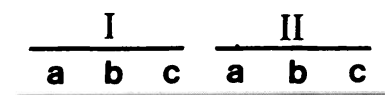

IFN-

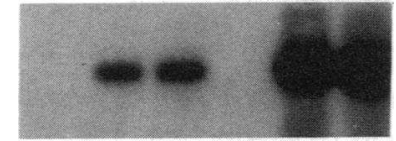

IL-10

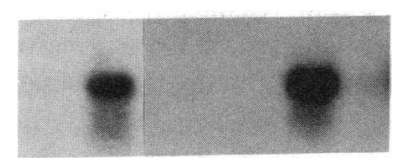

Figure 5. Cytokine mRNA patterns in PKDL patients. $(A) \mathrm{Re}-$ sponses of PBMC from two individual patients (I and II). Cytokine PCR was performed on cells cultured for $48 \mathrm{~h}$ with no stimulation $(a)$, with PHA for $24 \mathrm{~h}(b)$, or leishmania lysate for $48 \mathrm{~h}(\mathrm{C})$. (B) Cytokine profiles in a skin lesion biopsy of PKDL pa-

8

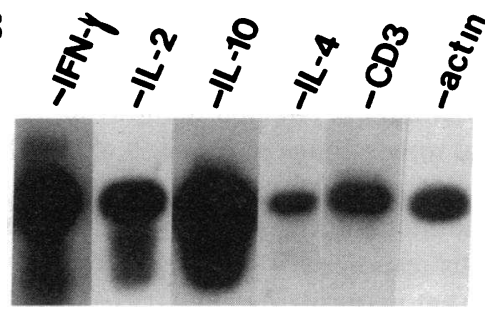
tient. PCR was performed on a parasitecontaining lesion of patient 1 of Fig. 5 . Normal uninfected skin biopsies had no detectable mRNA for the cytokines analyzed ( not shown). 
that activate macrophage killing mechanisms as well as cytokines that block activation of these cells can be critical in determining disease outcome.

Clinically, acute $L$. donovani or $L$. chagasi infections are typically nonhealing and are characterized by pancytopenia, splenomegaly, and lymphadenopathy. Certain immunological parameters, including high levels of parasite-specific antibodies and a lack of either parasite-elicited $\mathrm{T}$ cell proliferation or the production of biologically active IL- 2 and IFN- $\gamma$ may suggest that a Th2 cytokine response predominates during acute disease. Visceralized L. major infections in BALB/c mice have parallels with human infections, both in terms of pathogenesis and immunological parameters. In these animals, IL-4 and IL10 are associated with susceptibility, most evident by the prevention of disease by treatment with anti-IL-4 mAb (18). A lack of IFN- $\gamma$ production has been associated with disease progression in mice infected with L. major (18). However, in experimental acute $T$. cruzi infections, IFN- $\gamma$ production occurs in both susceptible and resistant mice, and it appears that the production of macrophage-inactivating cytokines, such as IL10 , is the determining factor in disease susceptibility (9).

Similarly, a dominant pattern of Th1 or Th2 cytokine production cannot be ascribed to human visceral leishmaniasis. The lymphoid tissues of patients with acute infections were characterized by both $\mathrm{Th} 1$ and $\mathrm{Th} 2$ cytokine production. In all patients tested, there was a clear pattern of IL-10 production during acute disease, and an absence of IL-10 after chemotherapy. However, IFN- $\gamma$ was present in both acute and treated patient samples, demonstrating that a Thl cytokine response was not absent in these individuals. This observation indicates that the presence of IL-10, shown to be produced by both Th1 and Th2 T cells in humans (29), rather than the absence of IFN- $\gamma$, is characteristic of uncontrolled human visceral leishmaniasis. Similarly, others have shown that susceptible BALB/ c mice also produce both Th1 and Th 2 cytokines during active leishmaniasis $(30,31)$. It is likely that cytokines such as IL-10 and IL-4, which down-regulate macrophage function, are important in the persistence of leishmania within their host cells. The detection of these cytokines in a PKDL lesion could help explain the persistence of parasites in these lesions even in the presence of IFN- $\gamma$ production.

In this study, we demonstrated that PBMC from acute visceral leishmaniasis patients had the ability to respond specifically to leishmania antigens by production of IL-10. This observation is important for several reasons. It suggests that acute patient $\mathrm{T}$ cells, generally believed to be unresponsive to leishmania antigen, can respond by producing IL-10. As opposed to solely measuring cytokine in tissues from acutely ill patients, this antigen specific IL-10 production lends particular relevance to the role of IL-10 in visceral leishmaniasis. The observation suggests further studies to define leishmania antigens capable of eliciting IL-10 production. It may also be speculated that genetically influenced cytokine production patterns could determine the course of human leishmania infection.

Several cell types can produce IL-10, including B cells, T cells, and macrophages $(6,32)$, and the cell source(s) of the IL-10 seen in visceral leishmaniasis patients remains to be established. It is possible that macrophage production of IL-10 in response to leishmania infection as well as IL-10 production by $T$ cells in response to leishmania antigens are both important for down-regulating $T$ cells responses. This role for IL-10 was supported by the finding that neutralizing anti-IL-10 $\mathrm{mAb}$ in- creased leishmania-specific proliferative responses of acute patient PBMC. In preliminary studies, we have observed elevated serum IL-10 levels in three of four acute visceral leishmaniasis patients tested, with a mean of 2.7 (SD 0.85) ng IL-10 per ml vs. undetectable levels (less than $62 \mathrm{pg} / \mathrm{ml}$ ) in healthy US and Sudanese controls, as determined by ELISA. Currently, nothing is known about the relevance of serum IL-10 in human disease, and these preliminary observations on serum levels must be considered of secondary importance to our findings of IL-10 in tissues and the active production of IL-10 by patient PBMC. However, the finding of IL-10 in patient sera, our demonstration of an antigen-specific antiproliferative effect of IL10 in vitro, and earlier reports that sera from visceral leishmaniasis patients contain substances capable of inhibiting proliferative responses $(33,34)$, may suggest a role for IL-10 in down-regulating in vivo immune responses. In this regard, others have reported increased serum IL-4 in visceral leishmaniasis patients (35).

In the present report, we have documented a correlation between IL-10 production and disease in a human parasitic infection. An association of increased IL-10 with pathogenesis has also been made in human leprosy (36). It appears that cytokines that decrease macrophage and $\mathrm{T}$ cell responses, such as IL-10, IL-4, and TGF- $\beta$ (37), may be critical in the pathogenesis of leishmania infections. Induction of these cytokines may be a key component of the virulence of macrophage parasites, and neutralization of these cytokines may represent a target for control of such infections.

\section{Acknowledgments}

We thank Dr. Robert Coffman and Kevin Moore, DNAX Research Institute, for serum IL-10 determinations and for human rIL-10 and anti-IL-10 mAb. We thank Karen Kinch for manuscript preparation. We gratefully acknowledge the expert technical assistance of Cari Brownell, Jeff Guderian, Steven Kuo, and Jackie Whittle.

This research was supported by the National Institutes of Health (grants AI-25038, AI-16282, and AI-16312), a Rockefeller Foundation Biotechnology Fellowship (to H. W. Ghalib), a Medical Research Council of Canada fellowship to (Y. A. W. Skeiky), and a National Institutes of Health fellowship (to D. M. Russo).

\section{References}

1. Murray, H. W., B. Y. Rubin, and C. D. Rothermel. 1983. Killing of intracellular Leishmania donovani by lymphokine-stimulated human mononuclear phagocyte: evidence that interferon gamma is the activating lymphokine. J. Clin. Invest. 72:1506-1509.

2. Fiorentino, D. F., M. W. Bond, and T. R. Mosmann. 1989. Two types of mouse helper T cell. IV. Th2 clones secrete a factor that inhibits cytokine production by Th1 clones. J. Exp. Med. 170:2081-2095.

3. Moore, K. W., P. Vieira, D. F. Fiorentino, M. L. Trounstine, T. A. Khan, and T. R. Mosmann. 1990. Homology of cytokine synthesis inhibitory factor (IL-10) to the Epstein Barr virus gene BCRFI. Science (Wash. DC). 248:12301234.

4. Fiorentino, D. F., A. Zlotnik, P. Vieira, T. R. Mosmann, M. Howard, K. W. Moore, and A. O'Garra. 1991. IL-10 acts on the antigen-presenting cell to inhibit cytokine production by Th1 cells. J. Immunol. 146:3444-3451.

5. De Waal Malefyt, R., J. Haanen, J. Spits, M. G. Roncarolo, A. te Velde, C. G. Figdor, and J. E. de Vries. 1991. Interleukin 10 (IL-10) and viral IL-10 strongly reduce antigen-specific human $\mathrm{T}$ cell proliferation by diminishing the antigen-presenting capacity of monocytes via down regulation of class II major histocompatability complex expression. J. Exp. Med. 174:915-924.

6. De Waal Malefyt, R., J. Abrams, B. Bennett, C. G. Figdor, and J. E. de Vries. 1991. Interleukin 10 (IL-10) inhibits cytokine synthesis by human monocytes: an autoregulatory role of IL-10 produced by monocytes. J. Exp. Med. 174:1209-1220. 
7. Ralph, P., I. Nakoinz, A. Sampson-Johannes, S. Fong, D. Lowe, H.-Y. Min, and L. Lin. 1992. IL-10, T lymphocyte inhibitor of human blood cell production of IL-1 and tumor necrosis factor. J. Immunol. 148:808-814.

8. Bogdan, C., Y. Vodovotz, and C. Nathan. 1991. Macrophage deactivation by interleukin 10. J. Exp. Med. 174:1549-1555.

9. Silva, J. S., P. J. Morrissey, K. H. Grabstein, K. M. Mohler, D. Anderson, and S. G. Reed. 1992. IL-10 and IFN- $\gamma$ regulation of experimental Trypanosoma cruzi infection. J. Exp. Med. 175:169-174.

10. Carvalho, E. M., R. S. Teixeira, and W. D. Johnson, Jr. 1981. Cell mediated immunity in American visceral leishmaniasis. Infect. Immun. 48:409-414.

11. Carvalho, E. M., R. Badaro, S. G. Reed, W. D. Johnson, and T. C. Jones. 1985. Absence of gamma interferon and interleukin-2 production during active visceral leishmaniasis. J. Clin. Invest. 76:2066-2069.

12. Sacks, D. L., S. Lata Lal, S. N. Shvivastava, J. Blackwell, and F. A. Neva. 1987. An analysis of T cell responsiveness in Indian Kala-azar. J. Immunol. 138:908-913.

13. Manson-Bahr, P. E. C., R. B. Heish, and P. C. C. Garnham. 1959. Studies in leishmaniasis in East Africa: the Montenegro test in Kala-azarin Kenya. Trans. R. Soc. Trop. Med. Hyg. 53:380-383.

14. Reed, S. G., R. Badaro, H. Masur, E. M. Carvalho, R. Lorenco, A. Lisboa, R. Teixeira, W. D. Johnson, and T. C. Jones. 1986. Selection of a skin test antigen for American visceral leishmaniasis. Am. J. Trop. Med. Hyg. 35:79-85.

15. Mosmann, T. R., H. Cherwinski, M. W. Bond, M. A. Giedlin, and R. L. Coffman. 1986. Two types of murine helper T cell clones. I. Definition according to profiles of lymphokine activity and secreted proteins. J. Immunol. 136:23482357

16. Cherwinski, H. M., J. H. Schumacher, K. D. Brown, and T. R. Mosmann 1987. Two types of mouse helper T cell clone. III. Further differences in lymphokine synthesis between Th1 and Th2 clones revealed by RNA hybridization, functionally monospecific bioassays, and monoclonal antibodies. J. Exp. Med. 166:1229-1244.

17. Sher, A., R. T. Gazzinelli, I. P. Oswald, M. Clerici, M. Kullberg, E. J. Pearce, J. A. Berzofsky, T. R. Mosmann, S. L. James, H. C. Morse III, and G. M. Shearer. 1992. Role of T-cell derived cytokines in the downregulation of immune responses in parasitic and retroviral infection. Immunol. Rev. 127:183-204.

18. Heinzel, F. P., M. D. Sadick, B. J. Holaday, R. L. Coffman, and R. M. Locksley. 1989. Reciprocal expression of interferon gamma or interleukin 4 during the resolution of progression of murine leishmaniasis. Evidence for expansion of distinct helper T cell subsets. J. Exp. Med. 169:59-72.

19. Heinzel, F. P., M. D. Sadick, S. S. Mutha, and R. M. Locksley. 1991. Production of IFN- $\gamma$, interleukin 2, interleukin 4 , and interleukin 10 by CD4+ lymphocytes in vivo during healing and progressive murine leishmaniasis. Proc Natl. Acad. Sci. USA. 88:7011-7015.

20. Reed, S. G. 1988. In vivo administration of recombinant interferongamma induces macrophage activation, and prevents acute disease, immune suppression, and death in experimental Trypanosoma cruzi infection. J. Immunol. 140:4342-4347.

21. Chomczynski, P., and N. Sacchi. 1987. Single step method of RNA isolation by acid guanidinum thiocyanate-phenol-chloroform extraction. Anal. Bio chem. 162:156-159.

22. Ehlers, S., and K. A. Smith. 1991. Differentiation of T cell lymphokine gene expression: the in vitro acquisition of T cell memory. J. Exp. Med. 173:2536.
23. Lewis, D. B., K. S. Prickett, A. Larsen, K. Grabstein, M. Weaver, and C. B. Wilson. 1988. Restricted production of interleukin 4 by activated human T cells. Proc. Nat. Acad. Sci. USA. 85:9743-9747.

24. Viera, P., R. de Waal Malefyt, M.-N. Dang, K. E. Johnson, R. Kastelein, D. F. Fiorentino, J. E. de Vries, M.-G. Roncarolo, T. R. Mosmann, and K. W. Moore. 1991. Isolation and expression of human cytokine synthesis inhibitory factor cDNA clones: homology to Epstein-Barr virus open reading frame BCRFF1. Proc. Natl. Acad. Sci. USA. 88:1172-1176.

25. Sambrook, J., E. F. Fritsch, and T. Maniatis. 1989. Molecular Cloning: A Laboratory Manual. Cold Spring Harbor Laboratory Press, Cold Spring Harbor, NY.

26. Feinberg, A. P., and B. Vogelstein. 1984. A technique for radiolabelling DNA restricted endonuclease fragments to high specific activity. Anal. Biochem. 137:266-267.

27. Reed, S. G., M. Barral-Netto, and J. A. Inverso. 1984. Treatment of experimental visceral leishmaniasis with lymphokine encapsulated in liposomes. J. Immunol. 132:3116-3119.

28. Murray, H. W., J. Stein, and K. Welte. 1987. Experimental visceral leishmaniasis: production of interleukin-2 and gamma interferon, tissue immune reactions, and response to treatment with interleukin-2 and gamma interferon. $J$. Immunol. 138:2290-2296.

29. Del Prete, G. F., M. De Carli, F. Almerigogna, M. G. Giudizi, R. Biagiotti, and S. Romagnani. 1993. Human IL-10 is produced by both type 1 helper (Th1) and type 2 helper ( Th2) T cell clones and inhibits their antigen-specific proliferation and cytokine production. J. Immunol. 150:353-360.

30. Morris, L., A. B. Troutt, E. Handman, and A. Kelso. 1992. Changes in the precursor frequencies of IL- 4 and IFN- $\gamma$ secreting CD4+ cells correlate with resolution of lesions in murine cutaneous leishmaniasis. J. Immunol. 149:27152721.

31. Chatelain, R., K. Varkila, and R. L. Coffman. 1992. IL-4 induces a Th2 response in Leishmania major-infected mice. J. Immunol. 148:1182-1187.

32. O'Garra, A., G. Stapleton, V. Dhar, M. Pearce, J. Schumacher, H. Rugo, D. Barbis, A. Stall, J. Cupp, K. Moore et al. 1990. Production of cytokines by mouse B cells: B lymphomas and normal B cells produce interleukin 10. Int. Immunol. 2:821-832.

33. Wyler, D. J. 1982. Circulating factor from a Kala-azar patient suppresses in vitro anti-leishmanial T cell proliferation. Trans. R. Soc. Trop. Med. Hyg. 76:304-306.

34. Barral, A., E. M. Carvalho, R. Badaro, and M. Barral-Netto. 1986. Suppression of lymphocyte proliferative responses by sera from patients with American visceral leishmaniasis. Am. J. Trop. Med. Hyg. 35:735-742.

35. Zwingenberger, K., G. Harms, C. Pedrosa, S. Omena, B. Sandkamp, and S. Neifer. 1990. Determinants of the immune response in visceral leishmaniasis: evidence for predominance of endogenous interleukin 4 over Interferon- $\gamma$ production. Clin. Immunol. Immunopathol. 57:242-249.

36. Yamamura, M., K. Uyemura, R. J. Deans, K. Weinberg, T. H. Rea, B. R. Bloom, and R. L. Modlin. 1991. Defining protective responses to pathogens: cytokine profiles in leprosy lesions. Science (Wash. DC). 254:277-279.

37. Barral-Netto, M., A. Barral, C. E. Brownell, Y. A. W. Skeiky, L. R. Ellingsworth, D. R. Twardzik, and S. G. Reed. 1992. Transforming growth factor- $\beta$ in leishmania infection: An important parasite escape mechanism. Science (Wash. DC). 257:545-548. 